\title{
Physiological conditioning by electric field stimulation promotes cardiomyogenic gene expression in human cardiomyocyte progenitor cells
}

\author{
Aida Llucià-Valldeperas', Benjamin Sanchez², Carolina Soler-Botija ${ }^{1}$, Carolina Gálvez-Montón ${ }^{1}$, Santiago Roura ${ }^{1}$, \\ Cristina Prat-Vidal ${ }^{1}$, Isaac Perea-Gil ${ }^{1}$, Javier Rosell-Ferrer ${ }^{3}$, Ramon Bragos ${ }^{3}$ and Antoni Bayes-Genis ${ }^{1,4,5^{*}}$
}

\begin{abstract}
The optimal cell lineage for cardiac-regeneration approaches remains mysterious. Additionally, electrical stimulation promotes cardiomyogenic differentiation of stimulated cells. Therefore, we hypothesized that electrical conditioning of cardiomyocyte progenitor cells (CMPCs) might enrich their cardiovascular potential. CMPCs were isolated from human adult atrial appendages, characterized, and electrically stimulated for 7 and 14 days. Electrical stimulation modulated CMPCs gene and protein expression, increasing all cardiac markers. GATA-binding protein 4 (GATA4) early transcription factor was significantly overexpressed $(P=0.008)$, but also its coactivator myocyte enhancer factor 2A (MEF2A) was upregulated $(P=0.073)$ under electrical stimulation. Moreover, important structural proteins and calcium handling-related genes were enhanced. The cardioregeneration capability of CMPCs is improved by electrical field stimulation. Consequently, short-term electrical stimulation should be a valid biophysical approach to modify cardiac progenitor cells toward a cardiogenic phenotype, and can be incorporated into transdifferentiation protocols. Electrostimulated CMPCs may be best-equipped cells for myocardial integration after implantation.
\end{abstract}

\section{Introduction}

Cardiovascular diseases remain the leading cause of death in Western countries. Alternative strategies beyond current guidelines are actively sought to repair injured cardiac tissue, and stem cell-based therapies provide a promising path toward achieving this goal. In the past decade, progenitors from different origins have been studied for cardiacregeneration purposes; however, the optimal cell lineage remains elusive. Despite the existence of resident cardiac stem cells, such as human cardiomyocyte progenitor cells (CMPCs), the regenerative capacity of the heart is limited.

The therapeutic potential of CMPCs was outlined in a pivotal report by Smits et al. [1], who demonstrated that

\footnotetext{
* Correspondence: abayes.germanstrias@gencat.cat

${ }^{1}$ ICREC (Heart Failure and Cardiac Regeneration) Research Program, Head of Cardiology Service, Germans Trias i Pujol University Hospital, Carretera del Canyet s/n, 08916 Badalona (Barcelona), Spain

${ }^{4}$ Cardiology Service, Germans Trias i Pujol University Hospital, Badalona (Barcelona), Spain

Full list of author information is available at the end of the article
}

CMPCs exhibit a certain degree of in situ differentiation into cardiomyocytes, smooth muscle cells, and endothelial cells after intramyocardial injection in a postinfarcted model in mice. Cardiomyogenic differentiation has also been promoted in a cardiac-mimetic electrical stimulation model in vitro [2]. Accordingly, we hypothesized that the biophysical conditioning of CMPCs by electrical stimuli might enhance their cardiovascular potential and render them (once electrostimulated) fitting candidates for cardiac cell-therapy strategies.

In this study, we reported CMPCs isolation and characterization; we designed an electrostimulation protocol based on 2-millisecond pulses of $25 \mathrm{mV} / \mathrm{cm}$ alternating current, and evidenced gene and protein modulations after electric-field stimulation.

\section{Results}

CMPCs were precisely isolated from human adult atrial appendages after the clonogenic method, as previously 
described [3]. Cell-collection procedure was approved by the local Ethics Committee (Germans Trias i Pujol University Hospital Ethics Committee), and informed consent was obtained from all patients. The study protocol conformed to the principles outlined in the Declaration of Helsinki.

Subsequent characterization of CMPCs demonstrated a spindle-shaped, highly proliferating (duplication time: $1.5 \pm 0.3$ days) population of cells showing high Sca- 1 and CD105, low CD34, and undetectable CD14, CD45, and $\mathrm{CD} 133$ expressions (Figure 1A-C). A representative CMPC cardiac gene-expression pattern was also found under basal conditions (Figure 1D). In particular, MEF2A and GATA4 transcription factors were expressed, as well as $\alpha$-actinin, cardiac troponin I (cTnI), the calcium handlingrelated protein, sarcoplasmic endoplasmic reticulum $\mathrm{Ca}^{2+}$ ATPase 2 (SERCA2), and the cell-connection protein, connexin43 (Cx43). Baseline expression of the endothelial marker CD31 was also confirmed in control CMPCs at both gene and protein levels (Figure 1D,E). See Additional file 1 for detailed explanation of materials and methods.

Next, CMPCs were electrostimulated by using a custommade stimulation-unit setup, which comprised a monophasic programmable electrical device, an electrical isolation stage, two printed circuit boards, which enable the fast and robust connection of the electrodes, and a biocompatible polydimethylsiloxane silicone-patterned construct, designed to provide structural support to cells and electrodes (Figure 2A). Although pulses produced by the stimulator are monophasic, the transformer-based isolator creates a negative low-level exponential pulse after each square positive pulse, which ensures zero direct-current average voltage to avoid electrolysis.

The electrical-stimulation protocol consisted of submitting 30,000 seeded cells to 2-ms monophasic square-wave pulses of $25 \mathrm{mV} / \mathrm{cm}$ at $1 \mathrm{~Hz}$ (alternating current) for 7 and 14 days [4].

Electrical stimulation modulated CMPC gene and protein expression (Figure 2B,C). Figure 2B shows the fold change of the studied cardiac markers at 7 and 14 days. All cardiac markers increased their expression after 14 days of stimulation.

A statistically significant overexpression of GATA4 was observed $(P=0.008)$, but also MEF2A $(P=0.073)$ was upregulated under electrical stimulation. MEF2 proteins are recruited through their DNA-binding domains by the early transcription factor GATA4 to activate cardiac promoters. Both transcription factors are expressed in

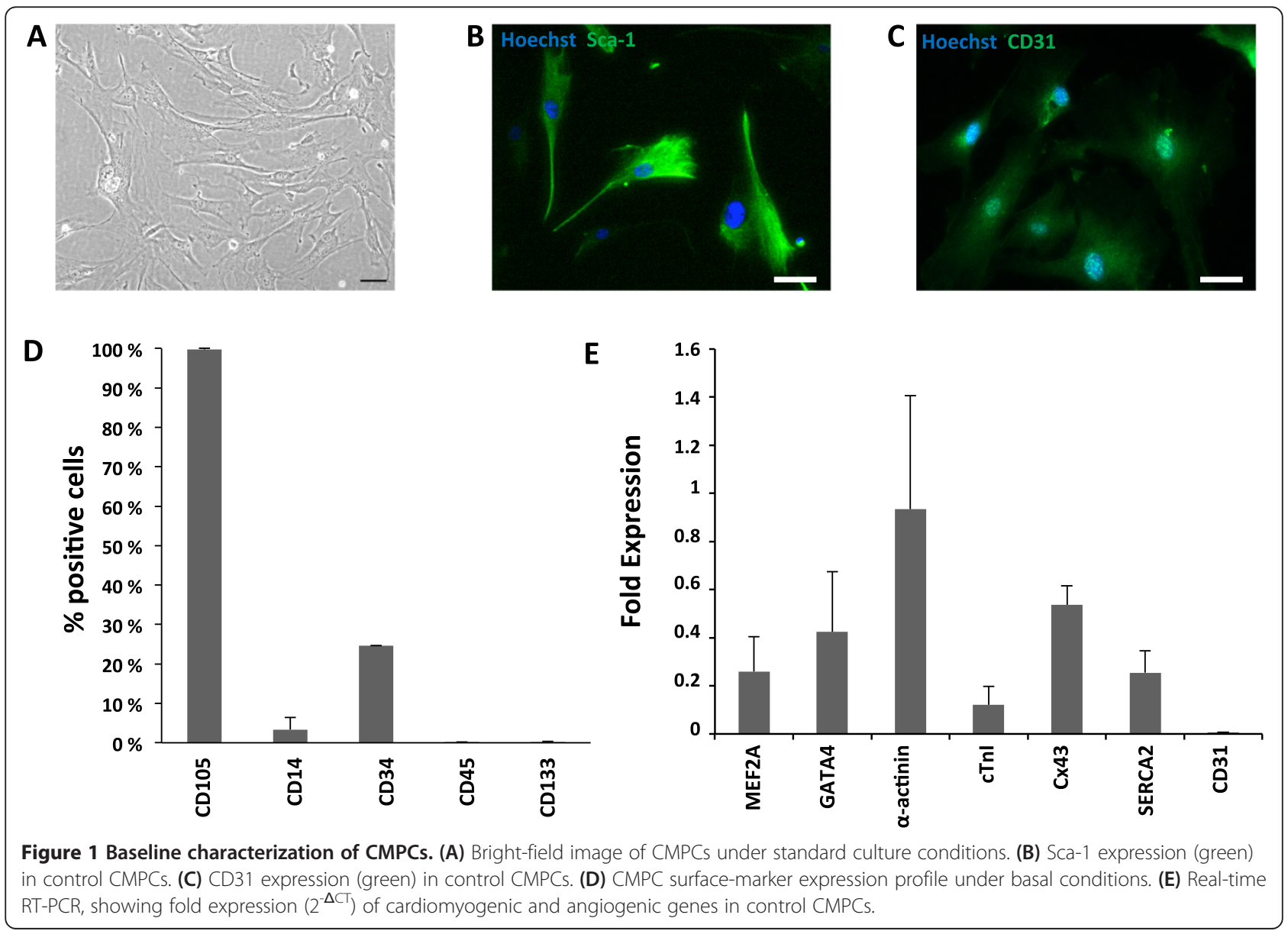




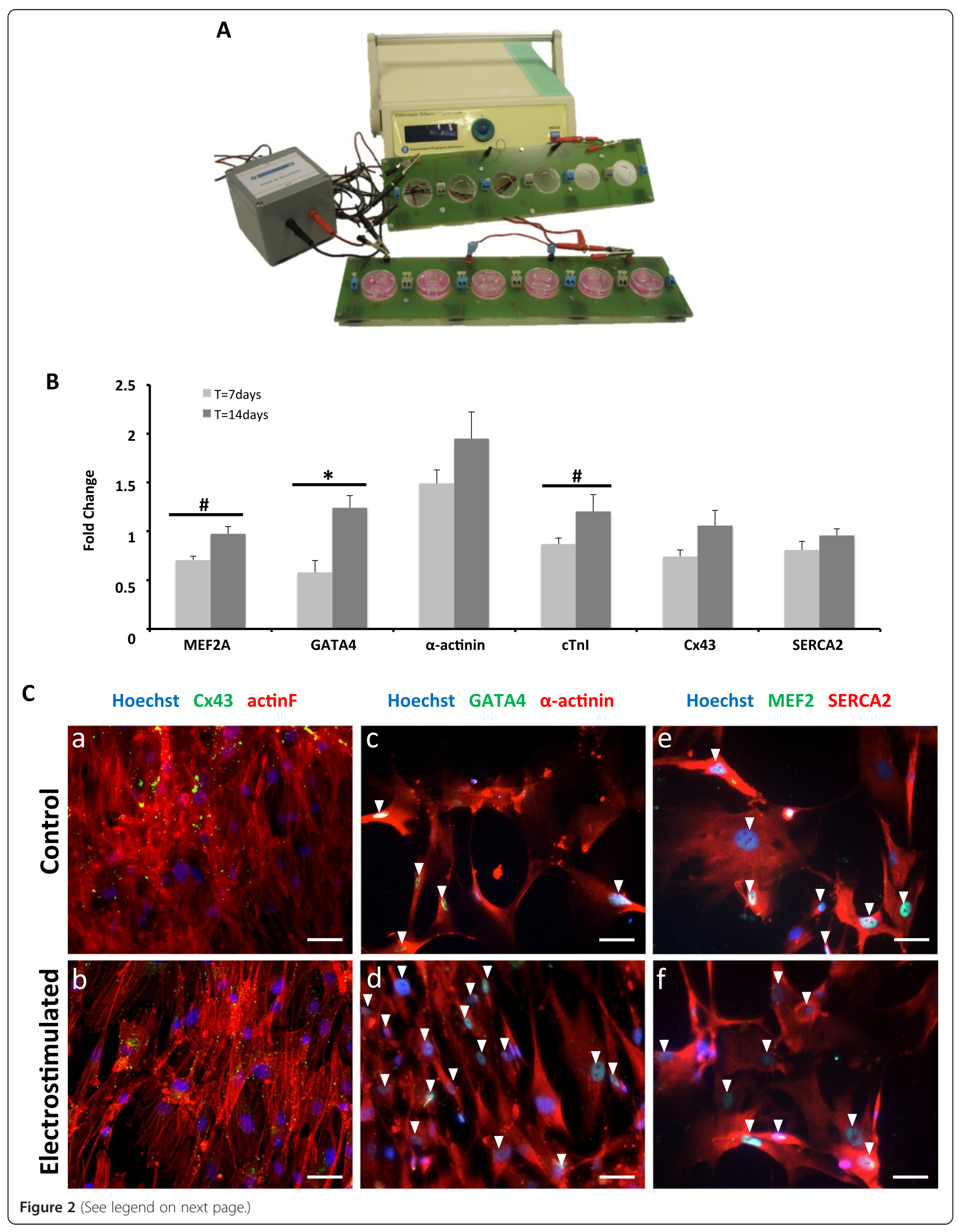


(See figure on previous page.)

Figure 2 Genetic and protein analysis after electrical stimulation. (A) Experimental setup for electric stimulation: the electric stimulator is connected to the printed circuit boards through an isolator stage. (B) Real-time RT-PCR of cardiac genes in electrically stimulated CMPCs, as compared with control, at 7 and 14 days. All RT-PCR values were analyzed in duplicate, normalized to GAPDH expression, and shown as fold-change mean \pm SEM; ${ }^{*} P<0.05$ and $\# P<0.10$, 7-day $(n=5)$ versus 14-day $(n=10)$ stimulation. (C) Actin fibers (red) and Cx43 (green), sarcomeric a-actinin (red) and GATA4 (green), and SERCA2 (red) and MEF2 (green) expressions in control $(\mathbf{a}, \mathbf{c}, \mathbf{e})$ and electrostimulated $(\mathbf{b}, \mathbf{d}, \mathbf{f}) \mathrm{CMPC}$, respectively. Nuclei were counterstained with Hoechst 33342. Scale bars $=50 \mu \mathrm{m}$. Arrowheads, positive staining of nuclear proteins (MEF2 and GATA4).

the developing heart and have similar genetic expression patterns after electrical stimulation.

The presence of early transcription factors might conceivably enhance cardiac protein expression to achieve further a cardiomyocyte-like phenotype (for example, gap junctions for electrical coupling, and sarcomeric proteins for mechanical contraction) (Figure 2Ca-f). Cx43 proteins form gap junctions, which are key elements for impulse propagation throughout the heart syncytium. Cx43 protein was observed in the cytoplasm, as well as at the plasma membrane, particularly in stimulated cells (Figure 2Ca,b), in which its expression was improved. Main structural proteins for the contractile apparatus, such as cTnI and $\alpha$-actinin, were also augmented $(P=0.093$ and $P>0.1$, respectively), although they do not show a striated pattern (Figure 2Cc,d). The absence of sarcomeres could suggest an early stage in the cardiomyogenic differentiation [5].

Additionally, SERCA2 protein was expressed in both conditions (Figure $2 \mathrm{Ce}, \mathrm{f}$ ), and was also slightly enhanced after 14 days of electrical stimulation $(P>0.1)$. SERCA2 proteins are intracellular pumps, which are located in the sarcoplasmic or endoplasmic reticula of muscle cells that are involved in the regulation of the cardiac contraction/relaxation cycle.

\section{Conclusions}

In sum, these data demonstrate that electric-field stimulation of CMPCs enhances cardiac gene expression. Gene modulation is translated to the protein level to promote CMPC phenotype differentiation. Short-term electrical stimulation appears to be a valid biophysical method to modify cardiac progenitor cells toward a cardiogenic phenotype, and can be included in transdifferentiation protocols. Electrostimulated CMPCs may be best-equipped for myocardial integration after transplantation.

\section{Additional file}

Additional file 1: Detailed description of materials and methods used in this study in the additional file.

\section{Abbreviations}

CMPCs: Cardiomyocyte progenitor cells; cTnl: cardiac troponin l; Cx43: connexin 43; GATA4: GATA-binding protein 4; MEF2: myocyte enhancer factor 2; SERCA2: sarcoplasmic endoplasmic reticulum $\mathrm{Ca}^{2+}$ ATPase 2.
Competing interests

Financial competing interests: Patent PCT/EP2012061224.

\section{Authors' contributions}

ALV participated in the design of the study, carried out the experimentation, collected and interpreted the data, and wrote the first draft. BS participated in the design of the study, developed the electrostimulation device, and reviewed the manuscript critically. CSB participated in the design of the study, helped with the experimentation, assisted in data interpretation, helped to draft the manuscript, and reviewed it critically. CGM participated in the design of the study, performed the statistical analysis, and reviewed the manuscript critically. SRF participated in the design of the study, helped with the experimentation, and reviewed the manuscript critically. CPV participated in the design of the study, helped with the experimentation, and reviewed the manuscript critically. IPG participated in the design of the study, helped with the experimentation, and reviewed the manuscript critically. JRF participated in the design of the study, developed the electrostimulation device, and reviewed the manuscript critically. RB participated in the design and coordination of the study, developed the electrostimulation device, reviewed the manuscript critically, and has given final approval of the version to be published. ABG conceived of the study, participated in its design and coordination, assisted in data interpretation, reviewed the manuscript critically, and has given final approval of the version to be published. All authors read and approved the final manuscript.

\section{Acknowledgements}

The authors thank the patients who made this study possible and the members of the Department of Cardiac Surgery for their collaboration in obtaining human samples. This work was supported by grants from the Ministerio de Economía y Competitividad (SAF2008-05144-C02-01 and SAF2011-30067-C02-01), European Commission 7th Framework Programme (RECATABI, NMP3-SL-2009-229239), La Marató de TV3 (122232), Redes de Investigación del Instituto de Salud Carlos III (Red de Terapia Celular (RD12/ 0019/0029) and Red de Investigación Cardiovascular (RD12/0042/0047)). We also appreciate support from the Fundació Privada Daniel Bravo Andreu. The funders had no role in study design, data collection and analysis, decision to publish, or preparation of the manuscript.

\section{Author details}

${ }^{1}$ ICREC (Heart Failure and Cardiac Regeneration) Research Program, Head of Cardiology Service, Germans Trias i Pujol University Hospital, Carretera del Canyet s/n, 08916 Badalona (Barcelona), Spain. 'Department of Neurology, Division of Neuromuscular Diseases, Beth Israel Deaconess Medical Center, Harvard Medical School, Boston, USA. ${ }^{3}$ Electronic and Biomedical Instrumentation Group, Departament d'Enginyeria Electrònica, Universitat Politècnica de Catalunya, Barcelona, Spain. ${ }^{4}$ Cardiology Service, Germans Trias i Pujol University Hospital, Badalona (Barcelona), Spain. ${ }^{5}$ Department of Medicine, Universitat Autònoma de Barcelona, Barcelona, Spain.

Received: 13 February 2014 Revised: 27 February 2014 Accepted: 21 July 2014 Published: 4 August 2014

\section{References}

1. Smits AM, van Laake LW, den Ouden K, Schreurs C, Szuhai K, van Echteld CJ, Mummery CL, Doevendans PA, Goumans MJ: Human cardiomyocyte progenitor cell transplantation preserves long-term function of the infarcted mouse myocardium. Cardiovasc Res 2009, 83:527-535.

2. Serena E, Figallo E, Tandon N, Cannizzaro C, Gerecht S, Elvassore N, Vunjak-Novakovic G: Electrical stimulation of human embryonic stem 
cells: cardiac differentiation and the generation of reactive oxygen species. Exp Cell Res 2009, 315:3611-3619.

3. Smits AM, van Vliet P, Metz CH, Korfage T, Sluijter JP, Doevendans PA, Goumans MJ: Human cardiomyocyte progenitor cells differentiate into functional mature cardiomyocytes: an in vitro model for studying human cardiac physiology and pathophysiology. Nat Protoc 2009, 4:232-243.

4. Llucià-Valldeperas A, Sanchez B, Soler-Botija C, Gálvez-Montón C, Prat-Vidal C, Roura S, Rosell-Ferrer J, Bragos R, Bayes-Genis A: Electrical stimulation of cardiac adipose tissue-derived progenitor cells modulates cell phenotype and genetic machinery. J Tissue Eng Regen Med 2013, (E-pub ahead of print), doi:10.1002/term.1710.

5. Qian L, Berry EC, Fu JD, leda M, Srivastava D: Reprogramming of mouse fibroblasts into cardiomyocyte-like cells in vitro. Nat Protoc 2013, 8:1204-1215.

doi: $10.1186 /$ scrt482

Cite this article as: Llucià-Valldeperas et al:: Physiological conditioning by electric field stimulation promotes cardiomyogenic gene expression in human cardiomyocyte progenitor cells. Stem Cell Research \& Therapy 2014 5:93.

\section{Submit your next manuscript to BioMed Central and take full advantage of:}

- Convenient online submission

- Thorough peer review

- No space constraints or color figure charges

- Immediate publication on acceptance

- Inclusion in PubMed, CAS, Scopus and Google Scholar

- Research which is freely available for redistribution 